\section{The contribution of reduced COVID-19 test time in control- ling the spread of the disease: A simulation-based approach}

\author{
Youness Frichi, ${ }^{1}$ Abderrahmane Ben \\ Kacem, ${ }^{2}$ Fouad Jawab, ${ }^{1}$ \\ Said Boutahari, ${ }^{1}$ Oualid Kamach, ${ }^{2}$ \\ Samir Chafik ${ }^{3}$ \\ ${ }^{1}$ Industrial Services and Technologies, \\ High School of Technology, Sidi \\ Mohamed Ben Abdellah University, Fez; \\ ${ }^{2}$ Laboratory of Innovative Technologies, \\ Abdelmalek Essaadi University, Tangier; \\ ${ }^{3}$ Multidisciplinary Research and \\ Innovation Laboratory, EMSI, \\ Casablanca, Morocco
}

\begin{abstract}
The novel coronavirus COVID-19 has known a large spread over the globe threatening human health. Recommendations from WHO and specialists insist on testing on a mass scale. However, health systems do not have enough resources. The current process requires the isolation of testees in the hospitals' isolation rooms for several hours until the test results are revealed, limiting hospitals' capacities to test large numbers of cases. The aim of this paper was to estimate the impact of reducing the COVID-19 test time on controlling the pandemic spread, through increasing hospitals' capacities to test on a mass scale. First, a discrete-event simulation was used to model and simulate the COVID-19 testing process in Morocco. Second, a mathematical model was developed to demonstrate the effect of accurate identification of infected cases on controlling the disease's spread. Simulation results showed that hospitals' testing capacities could be increased six times if the test duration fell from 10 hours to 10 minutes. The reduction of test time would increase testing capacities, which help to identify all the infected cases. In contrast, the simulation results indicated that if the infected population is not accurately identified and no precautionary measures are taken, the virus will continue to spread until it reaches the total population. Reducing test time is a vital component of the response to the COVID-19 pandemic. It is essential for the effective implementation of policies to contain the virus.
\end{abstract}

\section{Introduction}

\section{The novel coronavirus}

Currently, the world is experiencing an unprecedented health crisis that threatens global health. The severe acute respiratory syndrome coronavirus 2 (SARS-CoV-2) discovered in December 2019 in China, has spread globally, resulting in more than 4.9 million infections as of May 20, 2020. The coronavirus disease (COVID-19) affects the respiratory system and can lead to severe complications. It has caused a large number of deaths worldwide. Until now, there are no clinically approved vaccines or drugs available for COVID-19. ${ }^{1}$ The COVID-19 is transmitted via respiratory droplets and close contact with infected people. Its mean incubation period was estimated to be 3-7 days, ranging from 2 to 14 days, which expresses a long transmission period. Asymptomatic infected cases during the incubation period can transmit the SARSCoV2. ${ }^{1}$ According to epidemiological models, the virus could infect a considerable population unless strong measures are

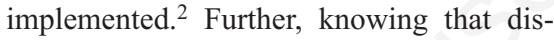
covering safe and effective vaccines or drugs requires, in optimistic scenarios, between 9 to 18 months, the only way to prevent health disasters is to control the virus's spread. ${ }^{2}$

One of the challenges facing hospitals is the massive influx of patients exceeding hospitals' capacities. In such situations, hospital logistics can contribute to establishing a balance between demand and capacity. ${ }^{3}$ It helps to ensure better flow synchronization, especially in exceptional situations that necessitate accurate resources sizing and planning. ${ }^{4,5}$ COVID-19 is currently submerging hospitals worldwide, with a massive influx of patients needing to be tested or hospitalized. Hospitals' capacities should be increased to meet this demand. However, hospitals' resources are limited. Hence, it is necessary to control the spread of the virus to avoid hospitals overwhelming. Scientists argue that faster tests to detect infected cases are effective ways to control the pandemic. Tests with reduced time would increase hospitals' testing capacities, allowing a significant proportion of the population to be tested and infected cases to be identified and isolated. The greater the ability to detect infections quickly, the higher the chance of limiting the coronavirus's spread. ${ }^{6}$ Therefore, we can assume that reducing the COVID-19 test time is a determinant key to controlling the pandemic's proliferation. This paper aims to assess and predict the impact of reducing the COVID-19 test time on controlling the spread of the virus (Figure 1). We first aim
Correspondence: Youness Frichi, Industrial Services and Technologies, High School of Technology, Sidi Mohamed Ben Abdellah University, $30000 \mathrm{Fez}$, Morocco.

Tel.: +212670144244.

E-mail: youness.frichi@usmba.ac.ma

Key words: COVID-19; test time; discreteevent simulation; mathematical model; simulation.

Acknowledgments: The authors would like to thank the editor and the reviewers for their valuable comments helping us to improve the article.

Contributions: YF conceptualized and designed the study, collected data, simulated the processes, analyzed and interpreted the results, developed and revised the manuscript. ABK participated in the simulation of the studied processes and results' interpreting. FJ conceptualized and designed the study, supervised data collection, and reviewed the manuscript. SB, OK, and SC participated in the study's conception and design and reviewed the manuscript.

Conflict of interests: The authors have no conflicts of interest to declare.

Availability of data and materials: All data generated or analyzed during this study are included in this published article.

Ethics approval and consent to participate: Not applicable.

Informed consent: Not applicable.

Received for publication: 30 May 2020.

Accepted for publication: 27 August 2021.

This work is licensed under a Creative Commons Attribution NonCommercial 4.0 License (CC BY-NC 4.0).

CC Copyright: the Author(s),2021

Licensee PAGEPress, Italy

Journal of Public Health in Africa 2021; 12:1455 doi:10.4081/jphia.2021.1455

at examining the effect of reduced test time on hospitals' testing capacities using discrete-event simulation. Second, through a mathematical model, we demonstrate how accurate identification of the infected population can control the spread of the virus. A case study is conducted in Morocco.

\section{COVID-19 tests and spread}

One step in the process of fighting COVID-19 that requires great scientific efforts is the testing stage. There are likely many people with undetected infection because testing capacities do not detect all 
infected people. ${ }^{2}$ There is an urgent need for increased capacity to test all cases. The hope is to have facilities with enough capacity to test the entire population. Then, individuals who test negative could resume ordinary life immediately, and infected people must be treated and isolated. ${ }^{6}$ Some countries like South Korea have succeeded in controlling the virus proliferation because they have adopted widespread testing. ${ }^{7}$ For the time being, there are two dominant ways to test for COVID-19: ${ }^{8}$ i) polymerase chain reaction (PCR) tests: this type of test is designed to detect the RNA of the SARS-CoV-2 virus, causing COVID-19. It is used to detect very recent infections. It has a high level of accuracy, but results take around a day to come through. Also, a professional technician is required to perform these tests and analyze the data, which is costly; ${ }^{9}$ ii) antibodies tests: this type is designed to detect whether the person previously had the virus. Extracted blood or plasma samples are analyzed, and results are typically ready within 10 minutes. However, these tests are less sensitive; they can produce more false positives and false negatives. Indeed, it is difficult to detect early infections; only 3-6 days post-infection patients can be detected. ${ }^{9}$

WHO recommended the PCR tests because of their accuracy. ${ }^{10}$ The antibodies tests are adequate in the recovery phase. Some researchers have suggested testing COVID-19 by chest computed tomography (CT), which is a non-invasive examination for the diagnosis of lung disease. It can be used as a rapid tool for suspected cases. ${ }^{11}$ However, the CT is nonspecific imaging manifestations of acute lung disease and is associated with various infectious. The use of CT as a diagnostic test is not recommended for routine tests. ${ }^{12}$

\section{COVID-19 in Morocco}

The first case COVID-19 in Morocco was confirmed on March 02, 2020. Since then, the government has implemented proactive and precautionary measures to mitigate the virus's propagation. The government has engaged and prepared civil and military healthcare services as well as the private health sector to receive and treat infected persons. Further, the Moroccan industry has made significant efforts to produce enough individual protective masks. Despite all efforts, the virus continues to spread. Official figures show that 106,004 cases were tested for COVID-19, from which 7133 are confirmed cases as of May 11, 2020. Morocco has considerably increased its testing capacity; nevertheless, the number of tests is relatively low due to cost and time constraints.

The PCR tests are used in Morocco. Only 13 laboratories are mandatory to test for COVID-19. The average testing capacity is 3000 tests per day. These capacity limitations do not allow determining the real number of infected cases.

\section{Forecasting and prediction tech- niques}

Since the purpose of this paper is to assess and predict the impact of reduced COVID-19 test time on hospitals' testing capacity and the control of the virus spread, an overview of forecasting methods is necessary to choose accurate methods.

The COVID-19 context is different: a massive influx of patients, lack of sufficient information as it is an unprecedented situation, and unpredictability of the virus's behavior. Qualitative and quantitative forecasting methods cannot be applied easily. Indeed qualitative methods are adequate when knowledge about the phenomenon is well-established. ${ }^{13}$ Quantitative methods require historical data about the phenomenon under study. ${ }^{14}$ Although forecasting methods have been widely applied in the medical field, ${ }^{15}$ they are not appropriate to forecast the COVID-19 spread.

Modeling and simulation techniques are alternative methods to make accurate predictions. ${ }^{16,17}$ These techniques can be categorized into two categories: Agent-Based Simulation (ABS) and Discrete-Event Simulation (DES). The ABS focuses on modeling autonomous agents that interact with each other in complex systems, while DES focuses on modeling systems' processes. ${ }^{18}$ In this study, we chose DES to model the COVID-19 test process because it is more appropriate for process modeling.

\section{Materials and Methods}

This research aims to assess and predict the impact of reduced test time on hospitals' testing capacity and, consequently, on controlling the virus's spread. First, DES is used to model the testing process and examine the impact of reduced test time on testing capacity. Second, a mathematical model is developed to understand how accurate identification of the infected population is crucial to control the coronavirus's spread.

\section{Discrete-event simulation}

The DES allows the modeler to examine the effect of modifying single entities within the system on the entire system. It has been widely used in health researches and proved to be an effective tool to predict the impact of health policies and strategies before their implementation. ${ }^{19}$ To build the DES model, we first collected data related to the patient pathway to get tested from publicly available sources, namely the Ministry of Health web site https://www.sante.gov.ma/Pages/Accueil.as px, official local media http://www.mapnews.ma/en/, and COVID-19 dedicated communication supports http://www.covidmaroc.ma/Pages/Accueil.aspx. Thirteen laboratories/hospitals are mandatory to perform the COVID-19 test in Morocco, with a total average capacity of 3000 tests per day. Figure 2 represents the testing process,

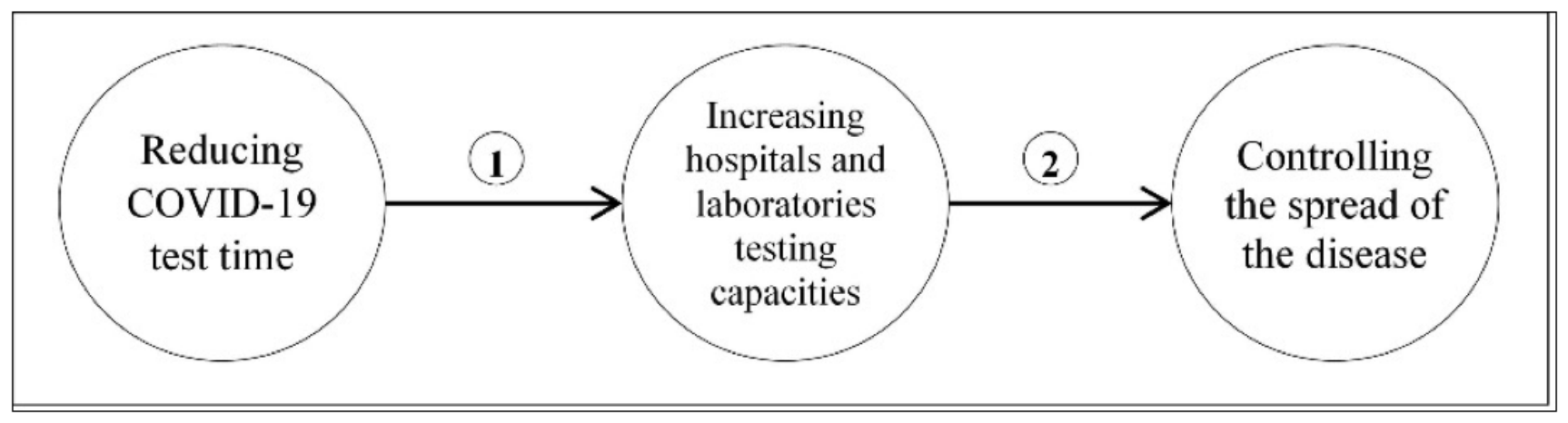

Figure 1. Impact of reduced COVID-19 test time on controlling the spread of the virus. 
which begins with a phone call to 141 received by emergency response centers that diagnose whether the case at hand is a potential case. Depending on the diagnosis's output, the emergency center decides whether to send an ambulance to transport the suspected case. Once at the hospital, the case is tested and isolated.

In the present study, we are mainly interested in the testing process, which starts with patients' arrival at the hospital reception for registration. They are then directed to a triage area, where the medical staff decides whether the patient needs to be tested. Patients with symptoms not related to COVID-19 are referred to adequate care units, while those presenting COVID-19 symptoms follow the next steps. Each potential COVID-19 case is tested and taken to an isolation room to wait for the results. The isolation room's current waiting time is around 6 to 10 hours, depending on the patient location as few laboratories can analyze COVID-19 tests. If the result is positive, the patient must immediately be taken to dedicated departments for treatment; otherwise, s/he leaves the testing pro- cess. This process was simulated on Arena software version 11 . We simulated different test times to demonstrate the impact of these times' length on hospitals' testing capacities.

\section{Mathematical model}

To demonstrate the crucial role of accurate identification of the infected population in preventing the virus's spread, we developed a mathematical model of the evolution of the infected population. Some assumptions are made for simplification: i) the infection is only dependent on the number of contacts between infected and uninfected people and on the infection probability; ii) no infection via touching surfaces or other objects is considered; iii) reported symptomatic infected cases are isolated; iv) no interaction between testees in the testing process; v) for there to be a new infection, two conditions must be met: an infected case $x_{\mathrm{i}}$ comes into contact with an individual $y_{\mathrm{i}}$, and the individual $y_{\mathrm{i}}$ has to be an uninfected case;

Let us note: $X_{j}$, the cumulative infected population on day $j ; Y_{j}$, the uninfected pop- ulation on day $j ; P T$, the total population:

$P T=X_{j}+Y_{j}$

$C$, the average number of contacts that each infected person has per day; $\alpha$, the infection probability that an uninfected person becomes infected when $\mathrm{s} /$ he has direct contact with an infected person; $\beta$, the percentage of the unreported and asymptomatic infected population. $\beta X_{j}$ is the number of unidentified infected people who are still contacting others because they are asymptomatic; $(1-\beta) X_{j}$ is the number of identified infected cases being isolated; $\rho_{j}$, the probability of a person to be uninfected:

$$
\rho_{j}=1-\left(\frac{X_{j}}{P T}\right)
$$

$\Delta X_{\mathrm{j}+1}$, the number of newly infected persons on day $j+1$

$\Delta X_{j+1}=\beta \times X_{j} \times C \times a \times \rho_{j}$

Equation (3) expresses the daily new infected persons. It results from unidenti-

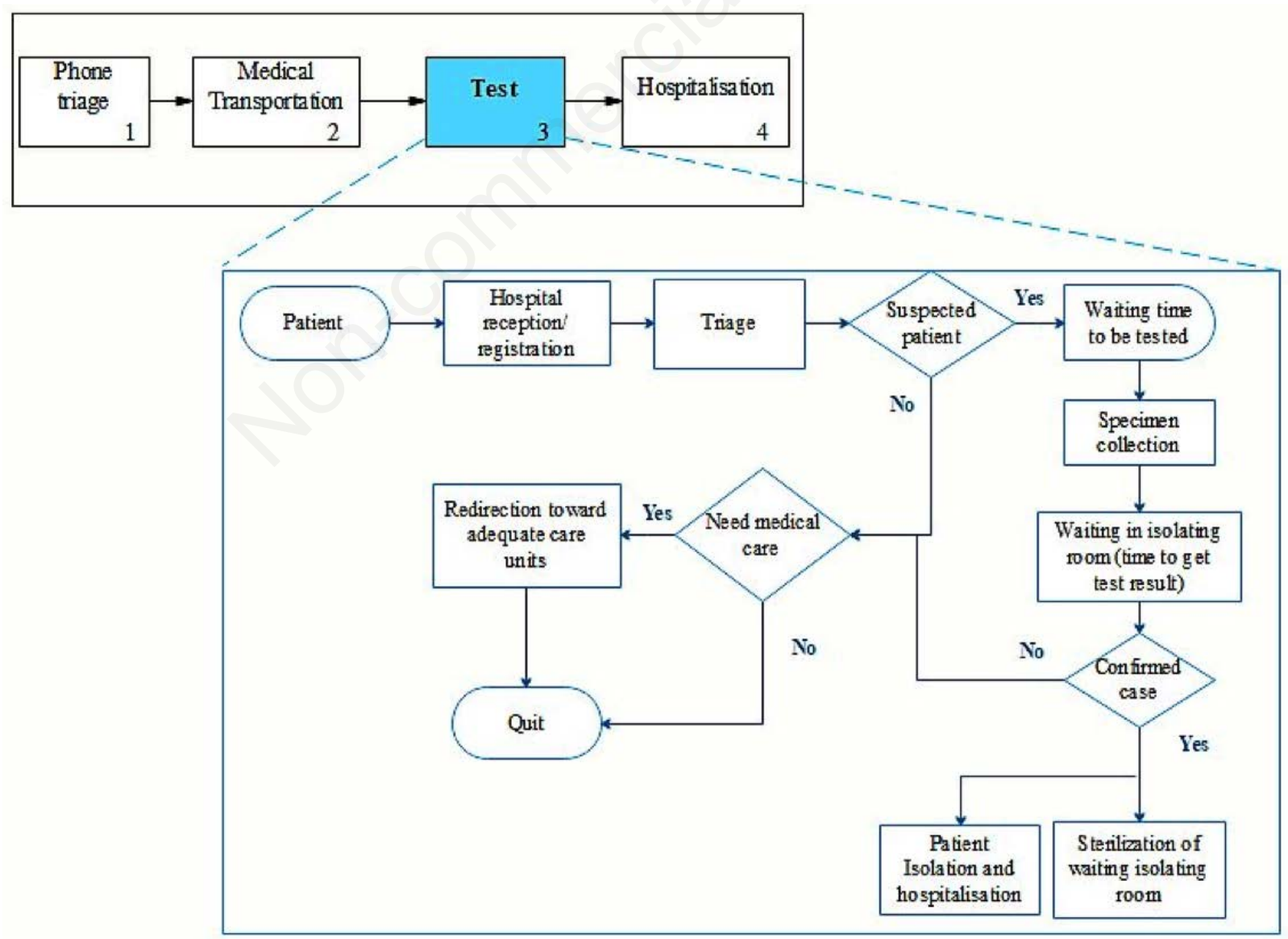

Figure 2. Mapping of patient trajectory for COVID-19 test in Morocco. 
fied infected cases, $\beta X_{j}$, who come into contact, at an effective contact rate $C$, with uninfected individuals with probability $\rho j$ and infection probability $\alpha$. For the sake of simplicity, we suppose that $\beta, C$, and $\alpha$ do not vary according to individuals or time. The cumulative number of infected persons on day $j+1$ can be expressed as:

$X_{j+1}=X_{j}+\Delta X_{j+1}$

$X_{j+1}=X_{j}\left(1+\beta \times C \times \alpha \times\left[1-\left(\frac{X_{j}}{P T}\right)\right]\right)$

From equation (5), $\beta, C$, and $\alpha$ are key parameters. If one of these parameters tends towards zero, then $X_{j+1}=X_{j}$, the number of infected cases is stable; the virus's spread is stopped. The first option $(\beta=0)$ signifies that all infected cases are identified. The second option $(C=0)$ means that there is no contact between infected and uninfected people. The third option $(\alpha=0)$ implies that the probability of infection is zero, which is unrealistic. However, it can be reduced if necessary measures of cleanliness and protection are undertaken.

The first option requires that the number of infected cases $X_{j}$ is accurately identified, which is only achievable by increasing the number of tests. Consequently, isolation measures could be applied so as there is no contact between uninfected and infected cases. Thus, the parameter $C$ is dependent on the parameter $\beta$ but not vice versa; if there is an unreported infected population, zero contact cannot be guaranteed.

If $X_{\mathrm{j}}$ is not accurately identified, then $\beta$ $\geq 0$ and $C \geq 0$, and consequently $X_{j+1} \geq X_{j}$. As long as the condition $X_{j+1} \geq X_{j}$ is met, the expression

\section{$\left(1+\beta \times C \times \alpha \times\left[1-\left(\frac{X_{j}}{P T}\right)\right]\right)$}

will be positive, then:

$$
\left[1-\frac{X_{j}}{P T}\right] \geq 0
$$

Or

$X_{j} \leq P T$

Consequently, the virus's spread will continue as long as the infected population is smaller than the total population.

\section{Results}

\section{ARENA simulation}

The DES model of Figure 2 was simulated on ARENA software (Figure 3). The simulation of the testing process is executed only for one laboratory/hospital. We assume that all 13 laboratories/hospitals have the same capacity of 230 tests per day. Patients' arrivals are considered to follow an exponential law. Each step's parameters were configured according to information collected from the Moroccan context:

Triage: is performed by hospitals' nurses, who decide whether the patient needs to be tested. We affected 15 nurses to the triage and supposed that $20 \%$ of patients need to be tested while $80 \%$ leave the testing process. The triage time is considered to be between 10 and 15 minutes for each patient.

Waiting time before the test: as in every non-urgent medical situation, patients may wait before being tested. A waiting time of 15 minutes was retained.

Specimens collection: is performed by specialists who take specimens from patients. We assigned 15 physicians and 15 nurses to collect specimens, and supposed that this task takes between 10 and 20 minutes for each patient.

Waiting in the isolation room: the patient is isolated in a dedicated room until the revelation of the results. The used PCR tests require 4 to 6 hours, at which it should be added logistics time. Thus, different durations are simulated from 10 hours (the current necessary time) to 10 minutes (for high innovative tests), and we used 90 iso- lation rooms. Patients with positive results stay at the hospital for isolation and medical care. Isolation rooms where patients with positive results stayed are disinfected before receiving other patients.

All the steps mentioned above and parameters are considered only for one hospital/laboratory.

A simulation on ARENA software was performed while fixing other resources, as stated above. The shorter the test time, the higher the testing capacity (Figure 4 ). The current test process allows only testing 230 persons per day. If the test time is reduced to 10 minutes, the capacity will reach 1400 persons. Test times shorter than 90 minutes do not significantly increase the testing capacity because it depends on other resources, such as the number of physicians, nurses, and isolation rooms.

\section{Simulation of the mathematical model}

The mathematical model was coded and compiled on MATLAB R2018a. Some assumptions are made: PT is equal to 35,904,061; the Moroccan population; 20 at day $j=1, X_{\mathrm{j}}$ was fixed at 1 ; on the first day, there was just one infected person; $C$ is set at 10; every infected person has ten contacts per day. This estimation is based on other studies conducted in Morocco; ${ }^{21}$ the infection probability $\alpha$ is fixed at $40 \%$. This estimate is based on the study of Ferretti et $a l .{ }^{22}$ the parameter $\beta$ was estimated from a study conducted in China. ${ }^{22}$ The ratio of infectiousness of asymptomatic individuals to symptomatic individuals was found to be 0.1 .

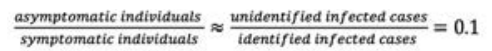

Or

$$
\frac{\beta X_{j}}{(1-\beta) X_{j}}=0.1
$$

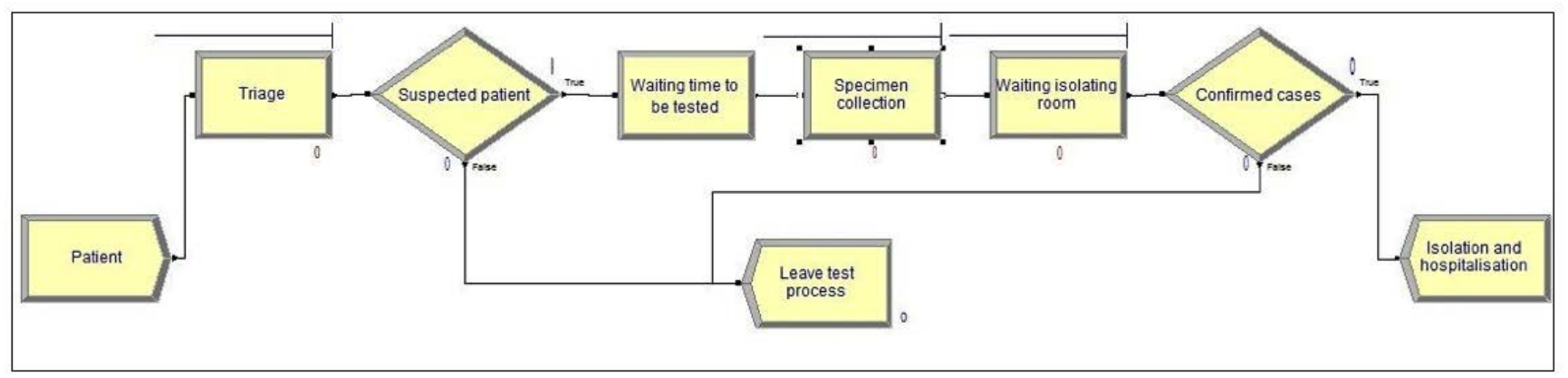

Figure 3. ARENA simulation model. 
The parameter $\beta$ can be estimated from equation (9), $\beta=0.09$. We believe that this parameter depends on each country's testing capacity. Thus, we considered assessing different $\beta$ values. Figure 5 shows the simulation results for $\beta €\{0.07,0.08,0.09,0.1$, $0.11\}$.

To contain the spread of coronavirus, the Moroccan government has implemented several measures to reduce interactions between people. On March 20, citizens are only allowed to go out to buy food or medicine and to work for those who perform vital functions. Non-essential services are closed. ${ }^{21}$ Besides, after $6 \mathrm{pm}$, no one is allowed to go out except for circumstances of necessity. These restrictive measures make it possible to reduce interactions between people. Hence, we have considered reducing the $C$ value to 5 . Also, since April 7 , wearing a protective face mask in public areas and workspaces has become an obligation for everyone. To accompany this obligation, the government has committed to ensuring the availability of masks at a reasonable price. This measure undoubtedly helps to reduce the probability of infection $\alpha$. Thus, we have considered reducing the $\alpha$ value to $20 \%$ (Figure 6).

Table 1 provides a recapitulative summary of the simulation results for different values of $\beta$ for given $C$ and $\alpha$ values. The peak size represents the maximum number of daily infected cases. The time of peak size is when the daily infected cases reach their maximum, and the total spread represents the time when the infected population reaches the total population.

Knowing that the coronavirus is highly contagious, the parameter $\alpha$ representing the infection probability cannot tend toward zero. It can only be reduced if precautionary measures are taken. In contrast, the parameter $\beta$ can be reduced or even tend toward zero if all infected cases are identified, which is only possible by increasing the number of tests. Concerning parameter $C$, it is dependent on $\beta$. Indeed, if all the infected population is identified and isolated, there will be no contact between infected and uninfected population.

\section{Discussion}

The $\mathrm{WHO}^{23}$ recommended a combination of measures to tackle the pandemic of COVID-19: very rapid diagnosis and immediate case isolation, rigorous tracking, and quarantine of close contacts. Particularly the WHO Director-General has insisted on testing, "We have a simple message for all countries: test, test, test". ${ }^{24}$ Increasing the

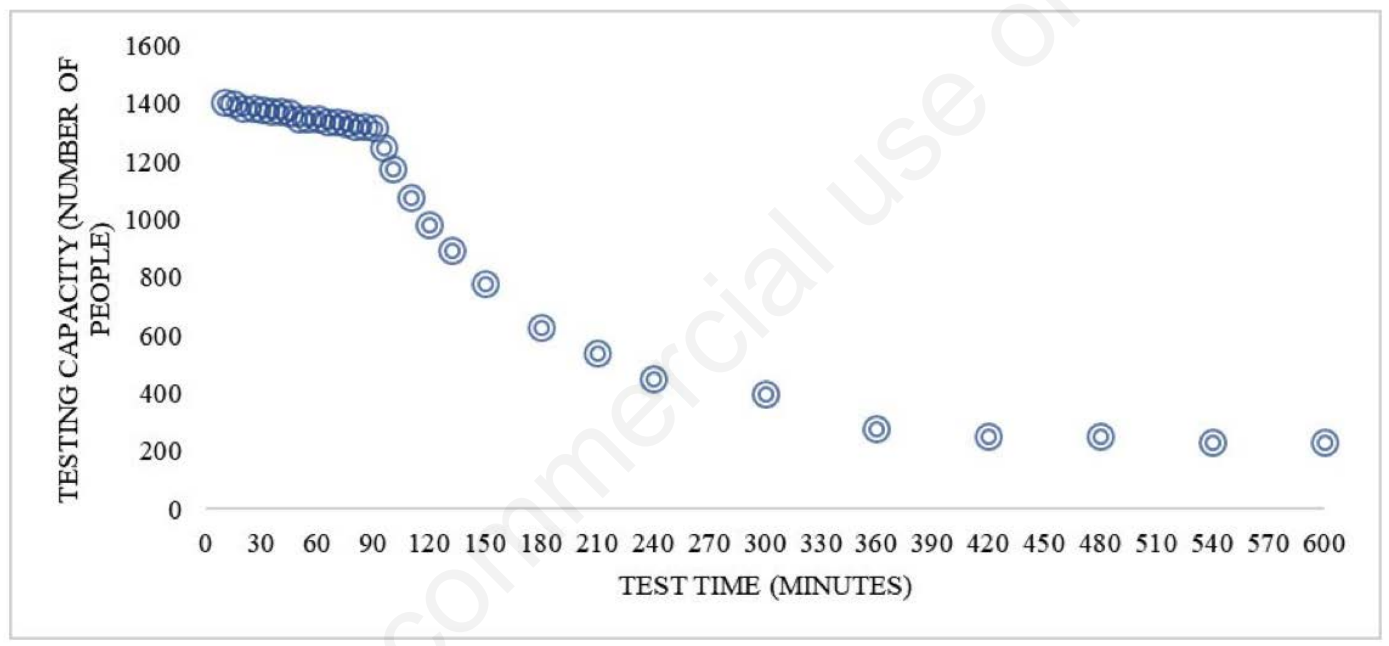

Figure 4. Variation of testing capacity according to the test time.
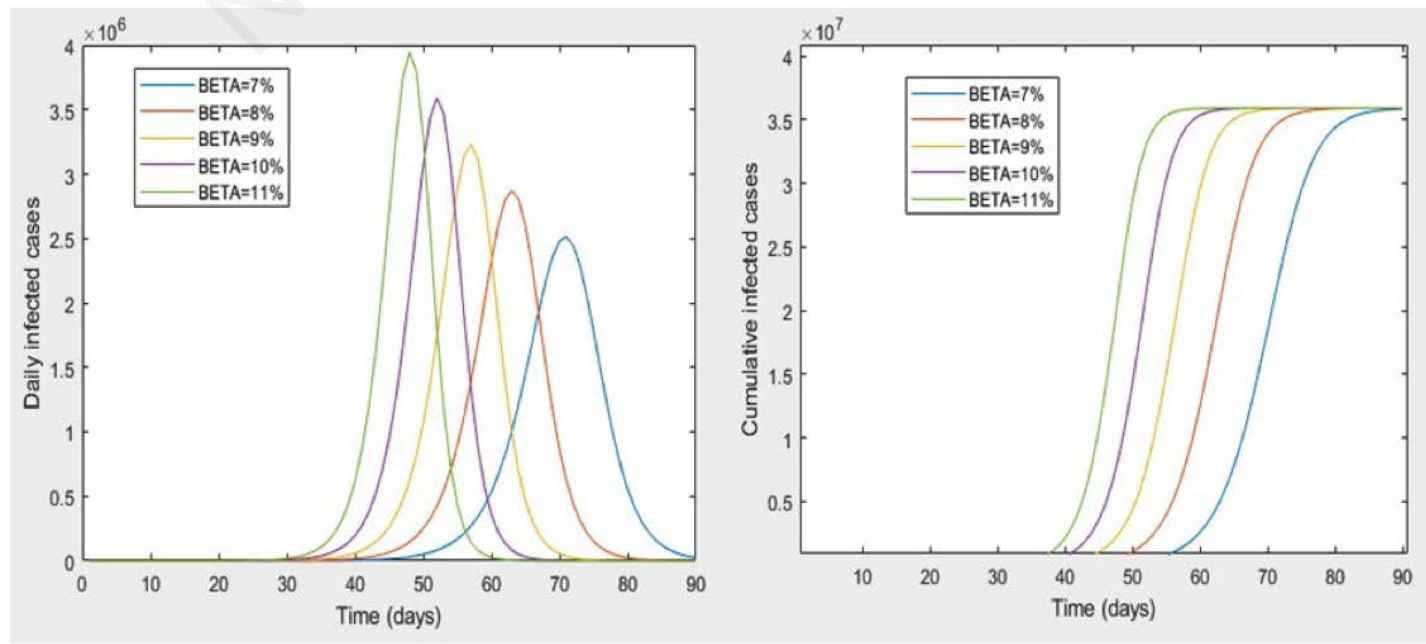

Figure 5. Simulation of daily and cumulative numbers of infected people cases for $\mathrm{C}=10$ and $\alpha=40 \%$. 
number of tests will allow countries to have a temporary clear picture of the virus's spread and implement the necessary policies. However, hospitals' testing capacities are limited. The PCR tests take around 4-6 hours plus logistics time, so results are provided after one day. During this time, testees are isolated in isolation rooms. Since the number of these rooms and other related resources is limited, the testing capacity is also limited. Thus, the number of tests per day is limited.

In this study, we first modeled the testing process using DES to evaluate the test time's impact on hospitals' testing capacities. Results showed that reduced test time increases the testing capacity, which allows testing of more cases. Second, we developed a mathematical model to highlight the critical parameters of the virus's spread.
Results from the simulation of the mathematical model prove the critical aspect of three parameters: the percentage of unidentified infected cases, the number of contacts that an infected person has per day, and the infection probability. The first parameter depends on the testing capacity. If the health system can test the whole population, the infected cases could be identified and isolated. Hence the virus will stop spreading. The second parameter is associated with the first one. The number of contacts between uninfected and infected cases can only be avoided if the infected population is identified accurately and isolated. Otherwise, the virus will continue to spread until the infected population reaches the total population. The third parameter is related to the infection probability. It is inevitable because the virus is proving to be highly contagious; nevertheless, it can be lowered through compliance with cleanliness and protection measures. The results of this research are in line with findings from other studies. Increasing the testing capacity was identified to be one of the core strategies adopted by resilient health systems to prevent widespread transmissions of COVID19. The experiences of Hong Kong, Singapore, and Japan are good examples. ${ }^{25}$

Innovation in testing is recognized to be necessary considering the constraints to test on a mass-scale. The innovation in test kits should have two main objectives: reduced test time and low testing cost. Other forms of innovation are also welcomed. For instance, in South Korea, the drive-through screening system does not require patients to go to hospitals. Patients are tested in their cars. Hence, isolation is kept in all testing

Table 1. Recapitulative results.

\begin{tabular}{|c|c|c|c|c|}
\hline $\mathrm{C}$ and $\alpha$ parameters & $\beta, \%$ & Peak size & $\begin{array}{l}\text { Time of peak size } \\
\text { (number of days after the March 2) }\end{array}$ & $\begin{array}{c}\text { Total spread } \\
\text { (number of days after the March 2) }\end{array}$ \\
\hline $\begin{array}{l}C=10 \\
\alpha=40 \%\end{array}$ & $\begin{array}{c}11 \\
10 \\
9 \\
8 \\
7\end{array}$ & $\begin{array}{l}3947000 \\
3589000 \\
3231000 \\
2872000 \\
2512000\end{array}$ & $\begin{array}{l}48 \\
52 \\
57 \\
63 \\
71\end{array}$ & $\begin{array}{l}56 \\
62 \\
69 \\
76 \\
87\end{array}$ \\
\hline $\begin{array}{l}C=5 \\
\alpha=40 \%\end{array}$ & $\begin{array}{c}11 \\
10 \\
9 \\
8 \\
7\end{array}$ & $\begin{array}{l}1974000 \\
1794000 \\
1612000 \\
1434000 \\
1257000\end{array}$ & $\begin{array}{c}88 \\
96 \\
106 \\
118 \\
133\end{array}$ & $\begin{array}{l}108 \\
121 \\
134 \\
148 \\
168\end{array}$ \\
\hline $\begin{array}{l}C=5 \\
\alpha=20 \%\end{array}$ & $\begin{array}{c}11 \\
10 \\
9 \\
8 \\
7\end{array}$ & $\begin{array}{l}987400 \\
897600 \\
806800 \\
717600 \\
628100 \\
\end{array}$ & $\begin{array}{l}167 \\
183 \\
203 \\
227 \\
258 \\
\end{array}$ & $\begin{array}{l}214 \\
234 \\
259 \\
289 \\
329\end{array}$ \\
\hline
\end{tabular}
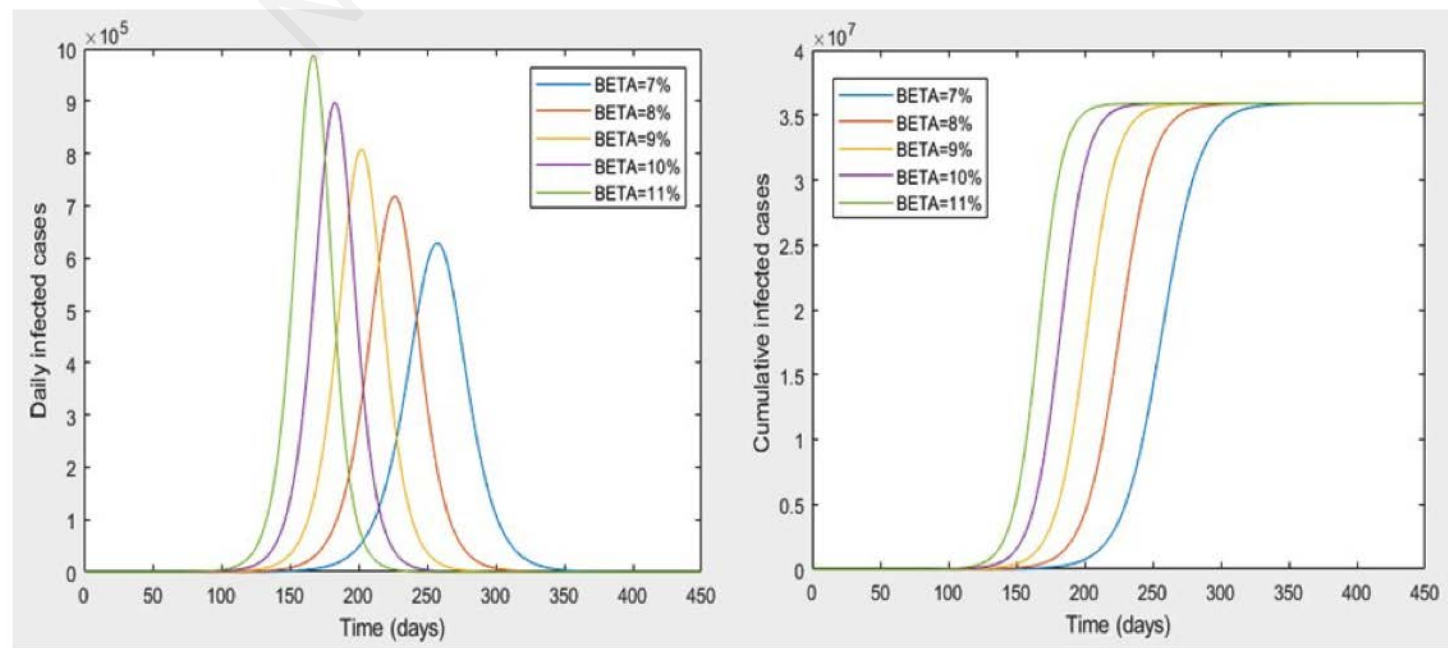

Figure 6. Simulation of daily and cumulative numbers of infected people cases for $C=5$ and $\alpha=20 \%$. 
processes, which excludes crossinfection. ${ }^{26}$

\section{Conclusions}

It is difficult to manage the crisis caused by the COVID-19 without knowing the real number of infections. The ability to rapidly detect the virus is of valuable help in slowing or even stopping its spread. Thus, massscale testing is encouraged, but limited resources are a barrier. In this paper, we showed, using simulations tools, the importance of tests with reduced time to increase the testing capacities and control the disease's spread. Reducing test time, on its own, will not stop the spread of the virus, but it will help keep the virus under control by preventing its transmission. The simulation of the testing process showed that test kits with shortened times could increase tremendously hospitals' testing capacities, which helps to identify all the infected cases. Further, the developed mathematical model demonstrates the effect of identifying the infected population accurately on containing the virus. Researchers are called open to focus their efforts on developing innovative testing procedures in terms of time and cost.

\section{References}

1. Li H, Liu S, Yu X, et al. Coronavirus disease 2019 (COVID-19): current status and future perspective. Int $\mathrm{J}$ Antimicrob Agents 2020;55:105951.

2. Salathé M, Althaus CL, Neher R, et al. COVID-19 epidemic in Switzerland: on the importance of testing, contact tracing and isolation. Swiss Med Wkly 2020;150:w20225.

3. Jawab F, Frichi Y, Boutahari S. Hospital logistics activities. International Conference on Industrial Engineering and Operations Management. Bandung, Indonesia; 2018.

4. Ben Kacem A, Kamach O, Chafik S, Ait Hammou M. A hybrid algorithm to size the hospital resources in the case of a massive influx of victims. Int J Electr Comput Eng 2020;10:1006-16.

5. Frichi Y, Jawab F, Boutahari S. Modeling the impact of hospital logistics on quality of care and patient satisfaction : results of a survey in three public healthcare facilities in Fez -
Morocco. J Ind Eng Manag 2020;13:296-320.

6. Peto J. Covid-19 mass testing facilities could end the epidemic rapidly. BMJ 2020;368:m1163.

7. Cohen J, Kupferschmidt K. Countries test tactics in "war" against COVID-19. Science 2020;367:1287-8.

8. Iacobucci G. Covid-19: What is the UK's testing strategy? Bmj. 2020;1222:m1222.

9. Bai H, Cai X, Zhang X. Landscape coronavirus disease 2019 test (COVID19 test) in vitro: a comparison of PCR vs Immunoassay vs Crispr-Based test. OSF Prepr. 2020.

10. WHO. Advice on the use of point-ofcare immunodiagnostic tests for COVID-19 [Internet]. WHO 2020. Available from: https://www.who.int/ news-room/commentaries/detail/ advice-on-the-use-of-point-of-careimmunodiagnostic-tests-for-covid-19

11. Li M. Chest CT features and their role in COVID-19. Radiol Infect Dis 2020;7:51-4.

12. Raptis CA, Hammer MM, Short RG, et al. Chest CT and Coronavirus Disease (COVID-19): a critical review of the literature to date. Am J Roentgenol 2020;215:839-42.

13. Chopra S, Peter M. Supply chain management: strategy, planning, and operation. Fifth edit. Pearson, 2013.

14. Frichi Y, Ben Kacem A, Jawab F, et al. Improving interhospital medical patient transportation in morocco: a forecasting collaborative approach. In: Euchi J, editor. Transportation, Logistics, and Supply Chain Management in Home Healthcare: Emerging Research and Opportunities. Pennsylvania, USA: IGI Global; 2020 p. 136-62.

15. Benkacem A, Frichi Y, Kamach O, et al. Proposal and validation of a model for predicting number of injuries due to road accidents in casablanca city. In: Ezziyyani M, editor. Advanced Intelligent Systems for Sustainable Development (AI2SD'2019). Lecture Notes in Networks and Systems. Springer, Cham; 2020 p. 371-82.

16. Sales JH. Epidemic covid mathematical model. Int J Latest Res Sci Technol. 2020;9:1-5.

17. Kanagarathinam K, Sekar K. Estimation of the reproduction number and early prediction of the COVID-19 outbreak in India using a statistical computing approach. Epidemiol Health 2020;42:e2020028.

18. Yin C, McKay A. Introduction to modeling and simulation techniques. ISCIIA and ITCA 2018 - 8th International Symposium on Computational Intelligence and Industrial Applications and 12th China-Japan International Workshop on Information Technology and Control Applications. 2018.

19. Zhang X. Application of discrete event simulation in health care: a systematic review. BMC Health Serv Res. BMC Health Serv Res 2018;18:1-11.

20. HCP. Site institutionnel du HautCommissariat au Plan du Royaume du Maroc [Internet]. 2020 [cited 2020 May 13]. Available from: https://www. hcp.ma/

21. Hammoumi A, Qesmi R. A first study on the impact of containment measure on COVID-19 spread in Morocco. medRxiv. 2020;2020.04.26.20080770.

22. Ferretti L, Wymant C, Kendall M, et al. Quantifying SARS-CoV-2 transmission suggests epidemic control with digital contact tracing. Science 2020;6936: eabb6936.

23. WHO. Report of the who-china joint mission on coronavirus disease 2019 (COVID-19) [Internet]. Report of The WHO-China Joint Mission on Coronavirus Disease 2019 (COVID19). 2020 . Available from: https://www.who.int/docs/defaultsource/coronaviruse/who-china-jointmission-on-covid-19-final-report.pdf

24. WHO. WHO Director-General's opening remarks at the media briefing on COVID-19-16 March 2020 [Internet]. 2020. Available from: https://www. who.int/dg/speeches/detail/who-director-general-s-opening-remarks-at-themedia-briefing-on-covid-19--16march-2020

25. Legido-Quigley H, Asgari N, Teo YY, et al. Are high-performing health systems resilient against the COVID-19 epidemic? Lancet 2020;395:848-50.

26. Kwon KT, Ko JH, Shin H, et al. DriveThrough Screening Center for COVID19: A safe and efficient screening system against massive community outbreak. J Korean Med Sci 2020;35:e123. 